\title{
A Novel Square-Spiral Strip Antenna
}

\author{
Ugur Saynak $^{* 1}$ and Alp Kustepeli ${ }^{2}$ \\ Izmir Institute of Technology, Dept. of Electrical and Electronics Eng. \\ Gulbahce Koyu, 35430 Izmir, Turkey \\ 1e-mail: ugursaynak@iyte.edu.tr Tel: +902327506235 \\ 2e-mail: alpkustepeli@iyte.edu.tr Tel: +902327506273
}

\begin{abstract}
A square-spiral strip antenna is designed considering the procedure used to obtain log-periodic antennas. Simulation results of the new design are compared with those of the well known and widely used Archimedean spiral antenna in terms of the frequency dependency. The effect of dielectric substrate used to support the antennas is also investigated.
\end{abstract}

Keywords-Frequency independent antennas, square-spiral antennas.

\section{INTRODUCTION}

Frequency independent antennas are used in applications requiring very wide bandwidths. Spiral, bi-conical and logperiodic antennas are generally classified as frequency independent antennas. Since spiral antennas can be constructed as planar structures and radiate circularly polarized waves, they are widely used when compared to the others.

A spiral antenna is designed usually using Archimedean or logarithmic geometries [1-7]. Although Archimedean spiral antennas may have wide operation bandwidths, they cannot be categorized as completely frequency independent according to Rumsey's scaling principle [8]. In cases where the spirals have small number of turns, Archimedean geometries are more frequency dependent compared to the logarithmic ones $[9,10]$. Spiral antennas are fabricated in round or square geometries. When the first radiation bands of the circular and square spiral antennas are examined one can see that the square spiral antennas have the advantage of operating with the same performances at lower frequencies $[1,5,11]$.

The purpose of this study is to design a square-spiral strip antenna which is more frequency independent than the Archimedean one. To have such an antenna a new geometry is obtained by employing the design method of log-periodic antennas. The new geometry for the antenna is given in Fig. 1. In this work the proposed antenna and equivalent size Archimedean spiral antenna are first analyzed and compared in free-space then a dielectric substrate is added to examine the effect of the support of antennas. The simulations are performed by using Finite Difference Time Domain method. Results are presented for a comparison especially in terms of frequency dependency of the fundamental antenna parameters.

\section{ANTENNA CONFIGURATION}

The equation defining the well know geometry of a round Archimedean spiral used in the antenna designs is given as

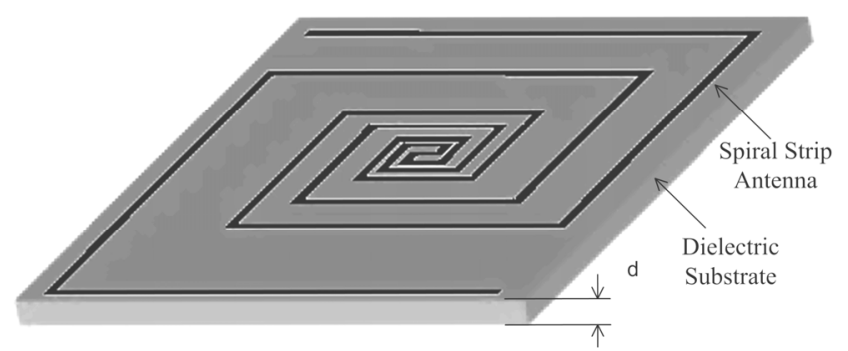

Figure 1. Proposed square-spiral strip antenna on dielectric substrate.

$$
\rho=a \varphi+b
$$

where $\rho$ and $\varphi$ are conventional polar coordinates, $a$ is the growth rate and $b$ is the starting point of the spiral curve on the $x$ axis. According to this equation, Archimedean spiral arms have a constant amount of growth rate at each turn. Square spiral antenna approximates the circular Archimedean spiral antenna in this manner. Therefore it always has the same distance between its arms.

Log-periodic antennas are designed by arranging the antenna elements, scaled by a constant $k$, operating at a single frequency. Log-periodic dipole antenna array (LPDA) is the simplest example for such periodic antennas. For LPDA, each dipole element and their location are determined according to a scale factor $k$ which is given as

$$
k=\frac{l_{n+1}}{l_{n}}=\frac{d_{n+1}}{d_{n}}=\frac{R_{n+1}}{R_{n}}
$$

where $l_{n}, d_{n}$ and $R_{n}$ are the length, the radius and the distance from a reference point of the $n^{\text {th }}$ dipole element, respectively.

Fig. 1 shows the general geometry of the proposed square spiral strip antenna designed considering the spiral and logperiodic antennas. The strip antenna is located in the $x-z$ plane. The dielectric substrate with a thickness $d$ used to support the antenna is also shown in the figure. The antenna is composed of two strip conductor arms, arranged similar to a log-periodic antenna. The first arm starts at the same starting point of the Archimedean antenna and expands with a constant ratio $k$. The second arm expands similar to the first one but it is arranged to be placed exactly between the two consecutive

\footnotetext{
*This work has been supported in part by EU-FP6 INCO "IYTE

Wireless" Project, Contract No: 017442.
} 


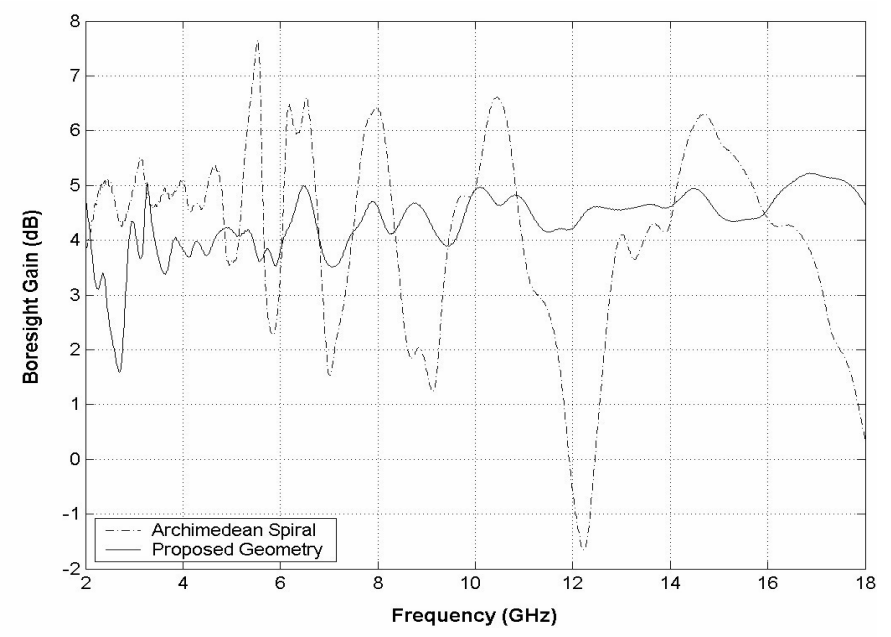

Figure 2. Gains for the antennas in free space.

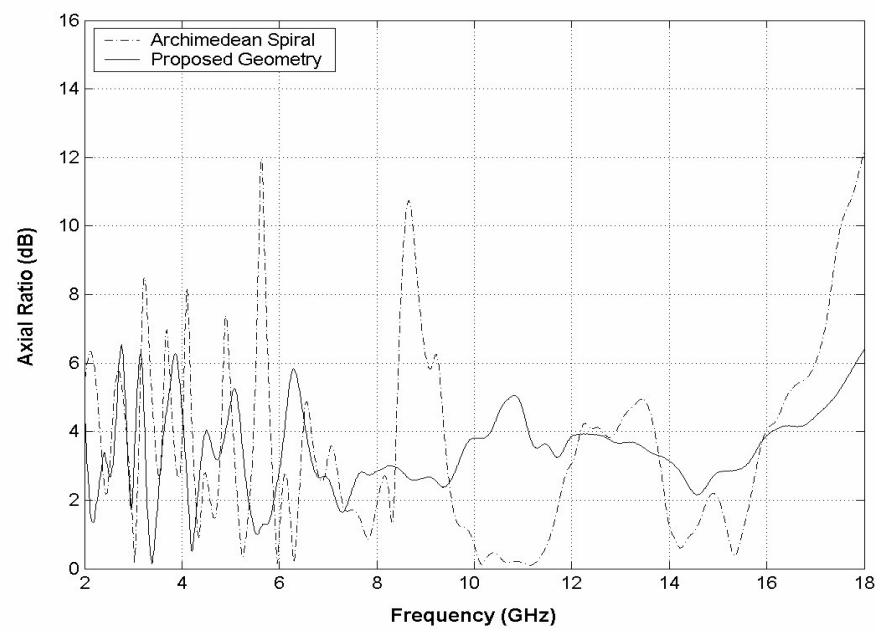

Figure 3. Axial ratios for the antennas in free space.

turns of the first arm. Consequently, because of this different design method, the new antenna is neither an approximation of the circular Archimedean spiral antenna nor an approximation of the logarithmic spiral antenna. Since some parts of the antenna are similar to the Archimedean spiral geometry and some parts are similar to the logarithmic spiral geometry, the proposed antenna can be interpreted as an approximation of both and can be called a hybrid Archimedean-logarithmic square spiral antenna.

\section{SimUlation RESUltS}

The simulation results for all of the antennas are obtained by using the finite difference time domain code XFDTD [12]. Archimedean and proposed antennas are first simulated in free space and then by adding a dielectric substrate to support the antennas. The antennas have 3 turns for both cases. They have an area of $8 \mathrm{~cm} \times 8 \mathrm{~cm}$ for the free space simulations and their feed gap is equal to $4.6 \mathrm{~mm}$ at their centers. For all of the simulations when the dielectric substrate is present its

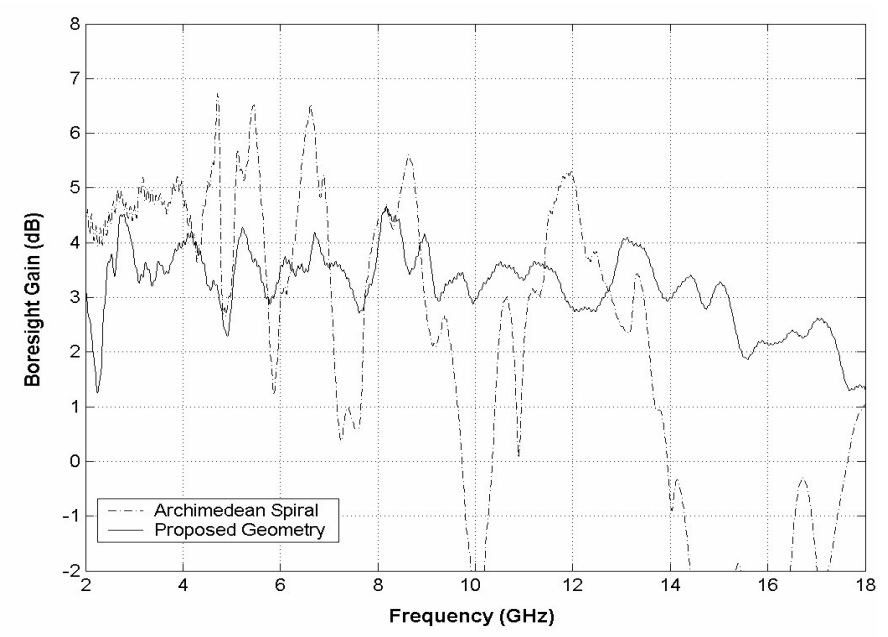

Figure 4. Gains for the antennas on dielectric substrate.

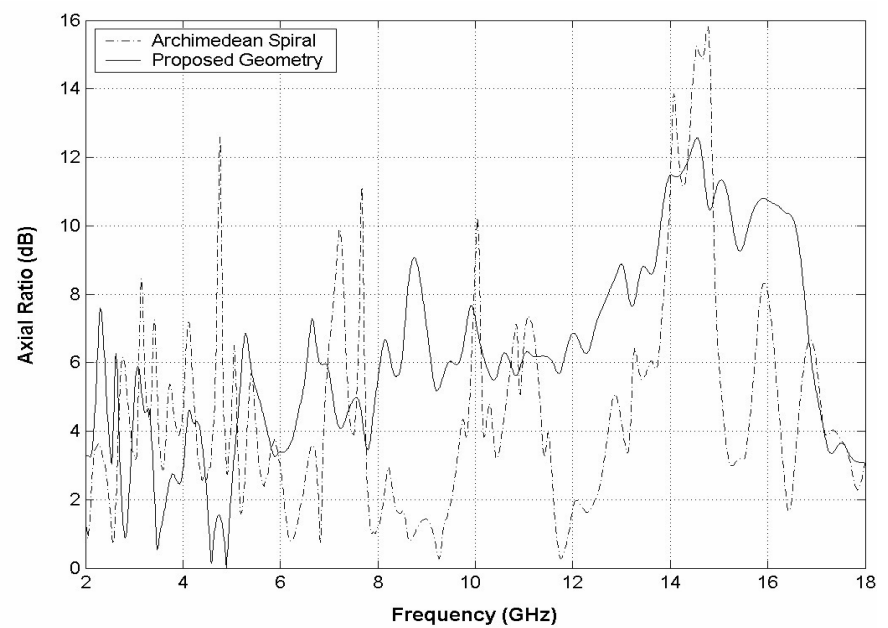

Figure 5. Axial ratios for the antennas on dielectric substraste.

thickness is equal to $1.6 \mathrm{~mm}$ and its dielectric constant is $\varepsilon_{r}=2.2$. The cell size in the analysis is chosen equal to the strip widths as $\Delta x=\Delta y=\Delta z=0.4 \mathrm{~mm}$ for free space simulations. When the antenna is supported by the dielectric substrate, the cell dimension $\Delta y$ is decreased to $0.2 \mathrm{~mm}$. The time step values are also chosen according to the cell sizes.

The gains and axial ratios for the antennas in free space are given in Fig. 2 and Fig. 3, respectively. As can be seen from the figures, the proposed antenna is more frequency independent than the Archimedean one. Extreme variations in the gain and axial ratio of the Archimedean spiral antenna are not present in the results of the proposed antenna. The results for the antennas with dielectric support are presented in Fig. 4 and Fig. 5. The effects of substrate on the antenna gains and axial ratios can be seen by comparing these figures with the previous ones. From figures Fig. 2 and Fig. 4 one can conclude that the gain of Archimedean antenna is much more affected than the proposed one especially at higher frequencies. One can also conclude that the dielectric support causes a contraction in 


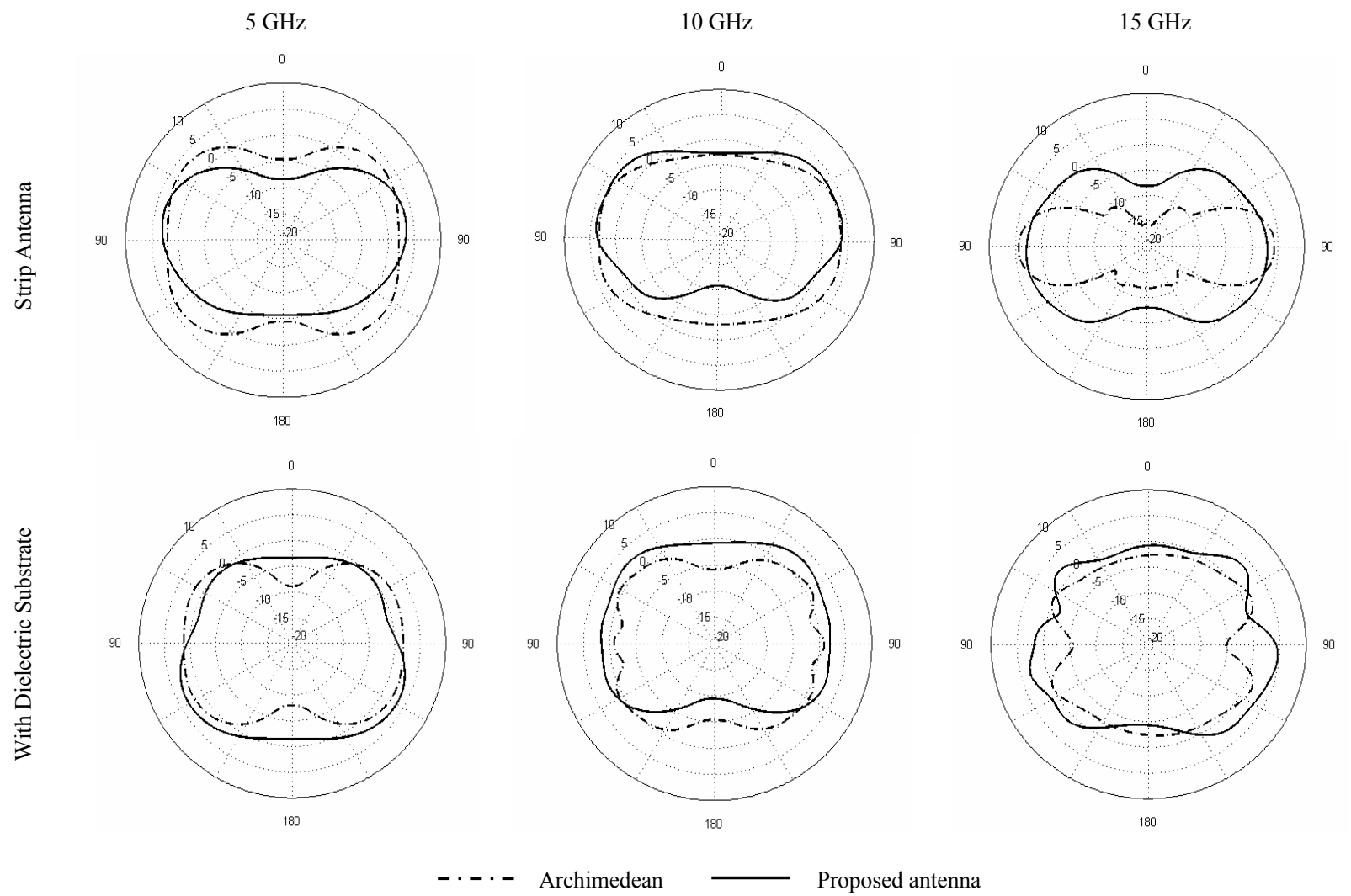

Figure 6. The radiation patterns of antennas in free space and on dielectric substrate $\left(\varphi=90^{\circ}, 0^{\circ}<\theta<180^{\circ}\right)$.

both of the gain and axial ratio values of the antennas in the frequency band.

The radiation patterns of antennas are presented in Fig. 5 at 5 $\mathrm{GHz}, 10 \mathrm{GHz}$ and $15 \mathrm{GHz}$ to give an idea about their frequency dependency. If the patterns are examined it can be seen that proposed antenna is less sensitive to frequency when compared to Archimedean spiral antenna. In addition, the radiation patterns of proposed antenna with or without the dielectric support are very similar to each other unlike the Archimedean spiral antenna. These results show that the proposed antenna has generally better characteristics when compared to Archimedean one.

To make a comparison in terms of the structure complexity, an Archimedean spiral antenna with 5 turns is also simulated in free space. The results obtained from the antenna and those of the proposed antenna configuration with 3 turns are shown in the Fig. 7 and Fig. 8. Although the gain of the Archimedean antenna is higher for lower frequencies, its variation at higher frequencies is much greater than the proposed antenna. One can also see that the performance of the proposed antenna is also better in terms of the axial ratio. Therefore the proposed antenna with simpler geometry performs better than the Archimedean antenna with 5 turns in terms of the frequency dependence of the gain and axial ratio.

\section{CONCLUSION}

In this study a novel antenna geometry is proposed and investigated for the square spiral strip antenna applications. The numerical analysis is performed by using the Finite Difference Time Domain method. The new antenna is compared with the well known Archimedean square spiral antenna in terms of the frequency dependence of the fundamental antenna parameters such as antenna gain, radiation pattern and axial ratio. Both of the antennas are also examined on dielectric substrates so that one can see the effect and the differences of the support material. The proposed antenna is also compared with a more complex Archimedean geometry obtained by increasing the number of turns.

From the simulation results, it can be seen that the frequency independence of the new antenna is generally better than the Archimedean antenna for all parameters considered. Same interpretation can also be made for the case when the dielectric substrate is present. Even the increase in the number of turns of the Archimedean spiral antenna does not improve its performance.

According to these results and interpretations, one can conclude that the proposed antenna can be used instead of a square Archimedean spiral antenna especially small number of turns considered for wide band applications. 


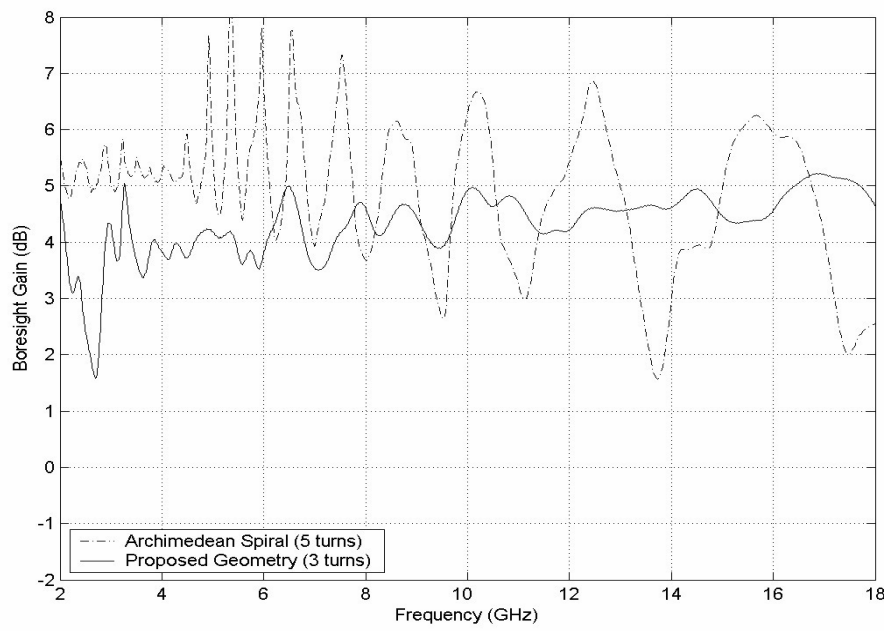

Figure 7. The gain comparison of proposed antenna with 3 turns and Archimedian spiral antenna with 5 turns.

\section{REFERENCES}

[1] Bawer, R., and Wolfe, J.J., "The Spiral Antenna,". IRE Int. Convention Record, New York, U.S.A., March 1960, pp. 84-95.

[2] J. Ely. C. Christodoulou and D. Shively, " Square Spiral Antennas for Wireless Applications,". IEEE NTC Conference, Orlando, FL., May 1995, pp. 229-232.

[3] K. Hirose, N. Saito, and H. Nakano, "A Cavity-Backed Spiral Antenna with an Unbalanced Feed System-Analyses Using FD-TD and Moment Methods,". Electronics and Communications in Japan, Part 1, Vol. 88, No. 1, 2005.

[4] Brown, E. R., Lee, A. W. M., Navi, B.S. and Bjarnason, J.E., "Characterization of Planar Self-Complementary Square-Spiral Antenna in the THz Region,". Microwave and Optical Tech. Lett., March 2006 , Vol. 48, No. 3, pp. 524-528.

[5] Kramer, B.A., Lee, M., Chen, C.C., and Volakis, J.L., "Design and Performance of an Ultrawide-Band Ceramic-Loaded Slot Spiral,". IEEE Trans. Antennas and Propagat, Vol. 53, No. 7, July 2005, pp. 21932199.

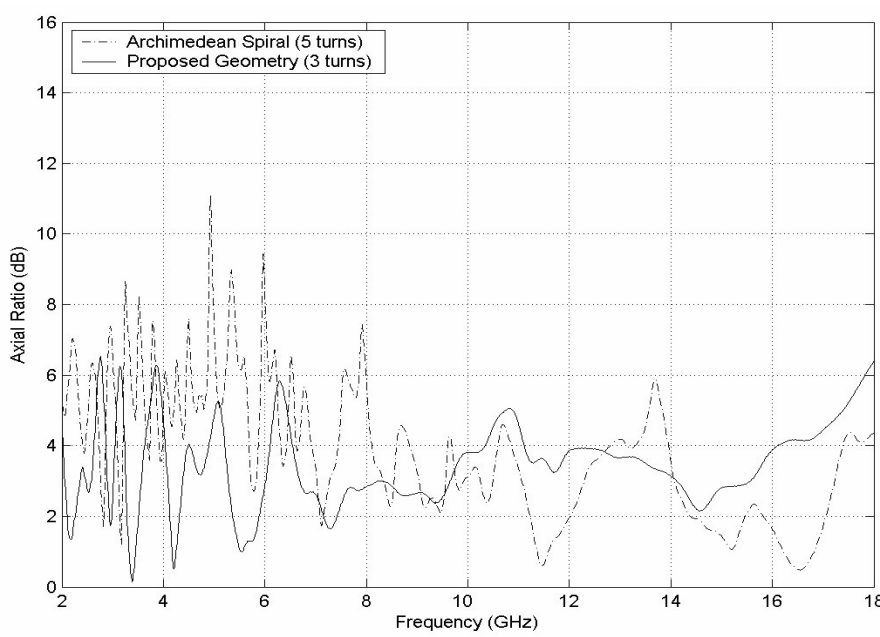

Figure 8. The axial ratio comparison of proposed antenna with 3 turns and Archimedian spiral antenna with 5 turns.

[6] Filipovic D.S., and Volakis J.L., "Broadband Meanderline Slot Spiral Antenna,". IEE Proc-Microw. Antennas Propagat., Vol. 149. 2 April 2002, pp. 98-104.

[7] Dyson, J.D., “The Equiangular Spiral Antenna,". IRE Trans. Antennas Propagat., 1959, 7, pp. 181-188.

[8] Rumsey, V.H., "Frequency Independent Antennas,". 1957 IRE National Convention Record, Pt. 1, pp.114-118.

[9] Balanis C.A., Antenna Theory-Analysis and Design, Wiley, New York, 1997.

[10] Kraus, J.D., Marhefka, R.J., Antennas, McGraw-Hill, New York, 2002.

[11] Kaiser, J.A., "The Archimedean two-wire spiral antenna,". IRE Trans. Antenna Propagat., Vol. AP-8, pp. 312-323, May 1960.

[12] XFDTD Bio-pro V6.2, Remcom inc., www.remcom.com. 